\title{
DoReMi: Context-based prioritization of linear motif matches
}

Many protein domains bind to short peptide sequences, called linear motifs. Data on their sequence specificities is sparse, which is why biologists usually resort to basic pattern searches to identify new putative binding sites for experimental follow-up. Most motifs have poor specificity and prioritization of the matches is thus crucial when scanning a full proteome with a pattern.

Here we present a generic method to prioritize motif occurrence predictions by using cellular contextual information. We take 2 parameters as input: the motif occurrences and one or more of the interacting domains. The potential hits are ranked based on how strongly the context network associates them with a protein containing one of the specified domains, which leads to an increased predictive performance. The method is available through a web interface at doremi.jensenlab.org, which allows for an easy application of the method. We show that this approach leads to improved predictions of binding partners for PDZ domains and the SUMO binding domain. This is consistent with the earlier observation that coupling sequence motifs with network information improves kinase-specific substrate predictions. 


\section{DoReMi: Context-based prioritization of linear motif matches}

3 Heiko Horn

4 NNF Center for Protein Research, University of Copenhagen, Denmark.

5 Niall Haslam

6 Complex and Adaptive Systems Laboratory, University College Dublin, Dublin, Ireland

7 Conway Institute of Biomolecular and Biomedical Science, University College Dublin, Dublin,

8 Ireland.

9 Lars Juhl Jensen

10 NNF Center for Protein Research, University of Copenhagen, Denmark.

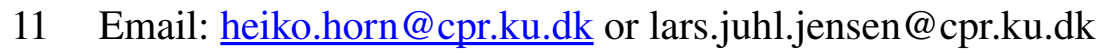

12

13

14

15

16

17

18

19

20

21

22

23

24

25

26

27

28

29

30

31

32

33

34

35

36

37

38

39

\section{Introduction}

Physical interactions among proteins play a crucial role in cellular signaling. There are two types of binding: domain-domain and domain-motif. For the latter type, a conserved domain interacts with a short peptide sequence (linear motif), which may be subject to post-translational modification (PTM) (Linding, 2010). Many proteins carry more than one domain, which allows them to bind to multiple different proteins and thereby act, for example, as scaffolds. Moreover, recent work has shown interdependence of PTM sites both with each other and with linear motifs (Minguez et al., 2012). Studying these binding sites and the domains that recognize them can thus help decipher the complex signalling networks in the cell.

The computational task of dealing with motifs can be split into two separate tasks: the discovery of novel, not known motifs and the search for new instances of already known motifs. The discovery of novel motifs is challenging, nonetheles a number of techniques have been developed to address this problem (Chou \& Schwartz, 2011; Davey et al., 2010; Lam et al., 2010). More recently structural approaches have been applied to the problem(Pathak et al., 2013), which similar to other tools rely on filtering out false positives in the discovery phase of motif identification. Physicochemical approaches have been developed to uncover patterns in the primary sequence that are characteristic of short peptide binding region(Dosztányi et al., 2009), and machine learning techniques employed to learn motifs from literature curated datasets(Lieber et al., 2010; Mooney et al., 2012; Nguyen Ba et al., 2012). Also, several resources exist that attempt to catalogue instances of linear motifs, incorporating manually curated and computational predictions of motifs (Dinkel et al., 2011; Mi et al., 2012; Sigrist et al., 2010). The discovery of both novel motifs and new instances is hindered by the problem of finding statistical significant matches. Many motifs are short peptide sequences and thereby appear in many different proteins by chance without representing a functional site (Obenauer et al., 2003).

When searching for new instances, all occurrences of a motif should thereby be screened for their biological validity. Based on the protein sequence, predictors for protein folding, structure and disorder can be used to filter for potential motifs (Davey et al., 2011). As functionally important sections of proteins tend to be conserved, further improvement can be gained by incorporating 
72 orthology information. Additionally, keyword annotations can help to distinguish the interesting proteins for the investigated processes (Ramu, 2003).

Further improvements can be achieved by integrating the cellular context of the interactions. As we try to filter for binding-associated motifs, the information about protein-protein interactions between the substrates and a protein containing the binding domain is an obvious approach. As long as this information is available, the question of biological relevance can be coupled to the question of co-localization and association; a binding site that will never be in proximity to the corresponding domain is biological not relevant in the studied context.

Previous work has shown that contextual information in the form of known physical interactions (Mi et al., 2012) or functional associations (Linding et al., 2007; Weatheritt \& Jehl, 2012) can improve motif based approaches. These methods are publicly available, but limit the query to a pre-defined set of motifs and interacting partners, which prevents their use for analysis of not already known motifs.

Here, we present DoReMi (Domain aided Regular Expression Mining), a tool that allows the user to specify any motif and the associated binding domain(s). By integrating the protein-protein association data into the scoring schema, we extend the usage from being a subsequently applied filter to a factor for ranking found potential instances. The algorithm is publicly available using the web interface at doremi.jensenlab.org.

\section{Materials \& Methods}

The algorithm takes a motif representation as input and scans a selection of representative sequences to find matching instances. To filter these hits, the score of a motif search gets combined with the protein-protein association scores derived from the STRING database to rank the occurrences (Franceschini et al., 2013). Previous work has demonstrated the utility of allowing both regular expressions (RegEx) and position specific scoring matrices (PSSM) to generate motif definitions (Haslam \& Shields, 2012); therefore we allow both of these as input as well as the result of any more advanced machine learning approach with a qualitative result.

The association scores are generated from the STRING confidence association scores. As the database only provides direct associations, indirect paths have to be calculated to cover also indirect interactions like e.g. scaffolding. Indirect interactions are computed by multiplying connecting edges on the path between the proteins of interest. To reduce the effect of overconnected nodes (hubs), we penalize for the connectivity by normalizing for the sum of all connecting edges of the node. The final score of an interaction can be described by the formula:

$$
\begin{gathered}
1+\sum_{i} i \\
\dot{i}^{\beta} \\
\dot{i}
\end{gathered}
$$$$
C\left(n_{n} \rightarrow n_{x}\right)-\left(C\left(n_{n-1} \rightarrow n_{n}\right)+C\left(n_{n} \rightarrow n_{n}\right)\right)
$$$$
\frac{C\left(n_{n-1} \rightarrow n_{n}\right)}{\dot{i}}
$$$$
\text { with } C\left(n_{n-1} \rightarrow n_{n}\right) \text { being the confidence score of }
$$ 
73 two nodes interacting and $\sum C\left(n_{n} \rightarrow n_{x}\right)$ being the sum of the confidence scores of all

74 connecting edges of $n_{n}$. The parameter $\beta$ was set based on our experience in the

75 NetworKIN project to a value of 0.34 .

76 After extending the network to cover indirect paths, the dataset covers a wide range of 77 associations between single proteins, each represented by a single score. This score can be 78 interpreted as the likelihood of two proteins interacting with each other.

79 The potential interaction partners are recruited by selecting all proteins containing the interacting 80 domain(s). To find these proteins, the Hidden-Markov-Model (HMM) representations provided by 81 the PFAM database are used to scan the whole STRING sequence database for matching 82 structures (Punta et al., 2012). For the scan, we use the HMMER3 package with default 83 parameters as stated by PFAM. These domain-protein associations are pre-calculated to later 84 increase the performance of the web service. All proteins carrying at least one of the interacting 85 domain(s) of interest are treated as potential interactors. In the case of multiple potential 86 interactors for one substrate, the highest scoring one is considered the most probable and its score 87 is used in the subsequent calculations.

88 The result from the PSSM search and the derived contextual network score are combined using a 89 weighting factor to be able to adjust the algorithm to different biological cases.

$$
\text { Final Score }=\text { Motif }^{1-\alpha} * \text { Association }^{\alpha}
$$

90

This weighting factor is used to account for the different impact the two factors have in the biological context. For example, the SUMO binding domain has a strongly conserved motif, leading to the assumption that the motif is sufficient to achieve good predictive performance. Conversely, the process of ubiquitination is well known for not having any preference for certain amino acids surrounding the modified residue, thus it is favorable to put more weight on the context information. If the motif search method only returns a binary value (e.g. regular expression returns only matches but no scores), DoReMi sorts the matches by the context network score alone.

To make the algorithm easily accessible and applicable, we developed a web service implementing the described workflow. The interface of this webservice allows for multiple different inputs for the motif finding part, which are subsequently enhanced with the precomputed network information.

\section{Results and Discussion}

To estimate the performance of our method, we selected two sets of binding sites, which have been previously been used for training of SUMOylation predictors (Matic et al., 2010; Ren et al., 2009). The family of SUMO (Small Ubiquitin-like Modifier) proteins comprises of multiple small members that are covalently attached to the proteins. As the name suggests, the proteins are similar to ubiquitin, and the whole enzymatic cascade is alike to the process of ubiquitination. SUMOylation is involved in functions like protein stability, nuclear-cytosolic transport, and transcriptional regulation. The SUMO binding motif can be split into 2 groups: The first motif is a strongly conserved one whereas the second is less restrictive. 
111 The older dataset from Ren et al. was used to calculate the PSSM and set the weighting factor $\alpha$. 112 We subsequently benchmarked the resulting predictor on the newer dataset by Matic et al., 113 removing all sites that have been already used in the training. To compare the approach of 114 creating a PSSM to a regular expression, we used the SUMOylation motif

$[\operatorname{VILMAFP}](K) . E$

115 from the Eukaryotic Linear Motif (ELM) database (Dinkel et al., 2011).

116 In addition, we trained a predictor based on the combined datasets and benchmarked its 117 performance by five-fold cross validation. The results of the partitioning were slightly better than 118 the results from training on one dataset and benchmarking on the other. This suggests that cross 119 validation gives a realistic performance estimate.

120 Given this, we also used cross validation to test the ability of DoReMi to predict binding partners for PDZ domains (Kim et al., 2012). PDZ domains are abundant in eukaryotes and eubacteria and commonly bind to the C-terminal end of proteins (Ponting, 1997). They are often associated with other domains like SH3 and therefor play an important role in the cell signaling(Zhang et al., 2011). The ELM database lists three different motifs, although they can be easily joined as they differ only in position four:

$$
\left.\begin{array}{l}
\ldots[D E] .[A C V I L F] \$ \\
\ldots[V L I F Y] .[A C V I L F] \$ \\
\ldots[S T] .[A C V I L F] \$
\end{array}\right\} \ldots[D E F I L S T V Y] .[A C V I L F] \$
$$

Overall, the motif is relatively loose and the $\mathrm{C}$-terminal location may be the strongest indicator for a real occurrence.

128 The combined set for SUMOylation contained 1063 sites while the publication by Kim et al. provided 367 instances. For the benchmarking, we pre-defined $\alpha$ to 0.4 and 0.8 for the SUMO and PDZ test sets respectively. We selected these values based on the conservation of the known sequences, as one can expect a greater influence of the cellular context, given the higher flexibility of the motif. Cross validation was repeated five times and the reported numbers are the median performance.

134 When selecting an appropriate score cut off by fixing the false positive rate ( 0.3 for SUMO, 0.15 for PDZ) we can show an improvement of the true positive rate by up to 15 percentage points for the PDZ domain and 10 for the SUMO binding domain. The smaller increase for the SUMO 137 domain is explained by the already good performance of the motif-based predictor, which leaves 138 less room for improvement. These examples use simple motif predictors; for comparison, we also 139 applied the context information to an already existing SUMO predictor (SUMOsp) to investigate 140 the possibility of improving on more advanced methods. We acknowledge that the result will be 141 influenced by the fact that this benchmark uses true positives on which the predictor got trained. 142 The shown improvement by using contextual information should be valid. Comparable to the first 143 examples, we see an improvement of the true positive rate by up to 10 percentage points, which 144 motivated the inclusion of this possibility into the web interface. A more detailed listing of the 145 performance numbers can be seen in Table 1. Although the overall performance is not significant 
better for some of the examples, in a real world scenario, the method performs better. The stated improvements mean, that if we would have a list of results and sort it by the final score, true positives are enriched in the beginning of this list. This is important, as the search of new instances of known motifs is mainly dominated by the task of filtering out false positives.

These results show that, although advanced methods like SUMOsp perform better than the basic approach of combining a RegEx or PSSM with contextual information, it can also be improved by the same approach. This shows the flexibility and versatility of this approach. Especially under the current state of lack of data that we see in the field of linear motif, it can be of great help in discriminating between true and false positive predictions. It also adds a non-sequence specific layer to many methods.

The web interface allows the user to easily apply the algorithm in a simple two-step process. The first step allows the user to define the potential binding sites. The first input type is specifying a regular expression; further details on the allowed format options are shown in Table 2. Alternatively, the user can provide a set of known binding peptides, which will be used to calculate a PSSM. Both the regular expression and the PSSM will be used to scan the human proteome for occurrences. As last option, the user can submit predictions from other motif search engines by providing identifier, position and score. These identifiers and positions have to be prior been mapped to the background sequence set used in DoReMi (STRING database version 9.05).

The second step allows for the selection of one or more potential binding domains. The interface allows the user to search for Pfam domains by their accession, id or description. From the list of search results, the user can select the relevant domains (Figure 1A). If necessary, multiple searches can be performed to select differently named domains.

The output page shows a brief summary of the analysis (Figure 1B). This includes plots of score distributions for each score (motif, network and combined score) to aid in the selection of an appropriate score cut-off for downstream analysis of the results after downloading.

\section{Conclusions}

Our work focused on making the integration of contextual information to enhance the search for motif occurrences into an easy to execute task. We show, that the addition of protein-protein interaction knowledge can improve the predictive performance of existing methods, even when doing it in a fully automated manner. This can be especially useful in the field of linear motifs; as for these, due to lack of data, advanced methods are not easy to develop. Further performance enhancements can potentially be reached when improving the domain associations in a more confined way.

For future enhancements, we plan to expand the usability of the method and web interface. Currently, only the human proteome is available; however, the method is in principle applicable to any fully sequenced organism. We thus consider expanding it to major eukaryotic model organisms. We are also working on integrating DoReMi with existing motif resources such as the ELM database. 
185

186

187

188

189

190

191

192

193

194

195

196

197

198

199

200

201

202

203

204

205

206

207

208

209

210

211

212

213

214

215

216

217

218

219

220

221

222

223

224

225

226

227

228

229

230

Chou, M. F., \& Schwartz, D. (2011). Biological sequence motif discovery using motif-x. Current Protocols in Bioinformatics / Editoral Board, Andreas D. Baxevanis ... [et Al.], Chapter 13, Unit 13.15-24. doi:10.1002/0471250953.bi1315s35

Davey, N. E., Haslam, N. J., Shields, D. C., \& Edwards, R. J. (2010). SLiMFinder: a web server to find novel, significantly over-represented, short protein motifs. Nucleic Acids Research, 38(Web Server issue), W534-9. doi:10.1093/nar/gkq440

Davey, N. E., Haslam, N. J., Shields, D. C., \& Edwards, R. J. (2011). SLiMSearch 2.0: biological context for short linear motifs in proteins. Nucleic Acids Research, 39(Web Server issue), W56-60. doi:10.1093/nar/gkr402

Dinkel, H., Michael, S., Weatheritt, R. J., Davey, N. E., Van Roey, K., Altenberg, B., Toedt, G., Uyar, B., Seiler, M., Budd, A., Jödicke, L., Dammert, M. a, Schroeter, C., Hammer, M., Schmidt, T., Jehl, P., McGuigan, C., Dymecka, M., Chica, C., Luck, K., Via, A., ChatrAryamontri, A., Haslam, N., Grebnev, G., Edwards, R. J., Steinmetz, M. O., Meiselbach, H., Diella, F., \& Gibson, T. J. (2011). ELM--the database of eukaryotic linear motifs. Nucleic Acids Research, 49(2), 1-10. doi:10.1093/nar/gkr1064

Dosztányi, Z., Mészáros, B., \& Simon, I. (2009). ANCHOR: web server for predicting protein binding regions in disordered proteins. Bioinformatics (Oxford, England), 25(20), 2745-6. doi:10.1093/bioinformatics/btp518

Franceschini, A., Szklarczyk, D., Frankild, S., Kuhn, M., Simonovic, M., Roth, A., Lin, J., Minguez, P., Bork, P., von Mering, C., \& Jensen, L. J. (2013). STRING v9.1: protein-protein interaction networks, with increased coverage and integration. Nucleic Acids Research, 41(Database issue), D808-15. doi:10.1093/nar/gks1094

Haslam, N. J., \& Shields, D. C. (2012). Profile-based short linear protein motif discovery. BMC Bioinformatics, 13(1), 104. doi:10.1186/1471-2105-13-104

Kim, J., Kim, I., Yang, J.-S., Shin, Y.-E., Hwang, J., Park, S., Choi, Y. S., \& Kim, S. (2012). Rewiring of PDZ domain-ligand interaction network contributed to eukaryotic evolution. PLoS Genetics, 8(2), e1002510. doi:10.1371/journal.pgen.1002510

Lam, H. Y. K., Kim, P. M., Mok, J., Tonikian, R., Sidhu, S. S., Turk, B. E., Snyder, M., \& Gerstein, M. B. (2010). MOTIPS: automated motif analysis for predicting targets of modular protein domains. BMC Bioinformatics, 11, 243. doi:10.1186/1471-2105-11-243

Lieber, D. S., Elemento, O., \& Tavazoie, S. (2010). Large-scale discovery and characterization of protein regulatory motifs in eukaryotes. PloS One, 5(12), e14444. doi:10.1371/journal.pone.0014444

Linding, R. (2010). (R)evolution of complex regulatory systems. Science Signaling, 3(127), eg4. doi:10.1126/scisignal.3127eg4

Linding, R., Jensen, L. J., Ostheimer, G. J., van Vugt, M. a T. M., Jørgensen, C., Miron, I. M., Diella, F., Colwill, K., Taylor, L., Elder, K., Metalnikov, P., Nguyen, V., Pasculescu, A., Jin, J., Park, J. G., Samson, L. D., Woodgett, J. R., Russell, R. B., Bork, P., Yaffe, M. B., \& Pawson, T. (2007). Systematic discovery of in vivo phosphorylation networks. Cell, 129(7), 1415-26. doi:10.1016/j.cell.2007.05.052

Matic, I., Schimmel, J., Hendriks, I. a, van Santen, M. a, van de Rijke, F., van Dam, H., Gnad, F., Mann, M., \& Vertegaal, A. C. O. (2010). Site-specific identification of SUMO-2 targets in cells reveals an inverted SUMOylation motif and a hydrophobic cluster SUMOylation motif. Molecular Cell, 39(4), 641-52. doi:10.1016/j.molcel.2010.07.026

Mi, T., Merlin, J. C., Deverasetty, S., Gryk, M. R., Bill, T. J., Brooks, A. W., Lee, L. Y., Rathnayake, V., Ross, C. a, Sargeant, D. P., Strong, C. L., Watts, P., Rajasekaran, S., \& 
Schiller, M. R. (2012). Minimotif Miner 3.0: database expansion and significantly improved reduction of false-positive predictions from consensus sequences. Nucleic Acids Research, 40(Database issue), D252-60. doi:10.1093/nar/gkr1189

Minguez, P., Parca, L., Diella, F., Mende, D. R., Kumar, R., Helmer-Citterich, M., Gavin, A.-C., van Noort, V., \& Bork, P. (2012). Deciphering a global network of functionally associated post-translational modifications. Molecular Systems Biology, 8(599), 599. doi:10.1038/msb.2012.31

Mooney, C., Pollastri, G., Shields, D. C., \& Haslam, N. J. (2012). Prediction of short linear protein binding regions. Journal of Molecular Biology, 415(1), 193-204. doi:10.1016/j.jmb.2011.10.025

Nguyen Ba, A. N., Yeh, B. J., van Dyk, D., Davidson, A. R., Andrews, B. J., Weiss, E. L., \& Moses, A. M. (2012). Proteome-wide discovery of evolutionary conserved sequences in disordered regions. Science Signaling, 5(215), rs1. doi:10.1126/scisignal.2002515

Obenauer, J. C., Cantley, L. C., \& Yaffe, M. B. (2003). Scansite 2.0: Proteome-wide prediction of cell signaling interactions using short sequence motifs. Nucleic Acids Research, 31(13), 3635-41. doi:10.1093/nar/gkg584

Pathak, S., Kundeti, V. K., Schiller, M. R., \& Rajasekaran, S. (2013). A Structure Based Algorithm for Improving Motifs Prediction. In A. Ngom, E. Formenti, J.-K. Hao, X.-M. Zhao, \& T. Laarhoven (Eds.), Pattern Recognition in Bioinformatics (Vol. 7986, pp. 242 252). Berlin, Heidelberg: Springer Berlin Heidelberg. doi:10.1007/978-3-642-39159-0

Ponting, C. P. (1997). Evidence for PDZ domains in bacteria, yeast, and plants. Protein Science : A Publication of the Protein Society, 6(2), 464-8. doi:10.1002/pro.5560060225

Punta, M., Coggill, P. C., Eberhardt, R. Y., Mistry, J., Tate, J., Boursnell, C., Pang, N., Forslund, K., Ceric, G., Clements, J., Heger, A., Holm, L., Sonnhammer, E. L. L., Eddy, S. R., Bateman, A., \& Finn, R. D. (2012). The Pfam protein families database. Nucleic Acids Research, 40(Database issue), D290-301. doi:10.1093/nar/gkr1065

Ramu, C. (2003). SIRW: A web server for the Simple Indexing and Retrieval System that combines sequence motif searches with keyword searches. Nucleic Acids Research, 31(13), 3771-4. doi:10.1093/nar/gkg546

Ren, J., Gao, X., Jin, C., Zhu, M., Wang, X., Shaw, A., Wen, L., Yao, X., \& Xue, Y. (2009). Systematic study of protein sumoylation: Development of a site-specific predictor of SUMOsp 2.0. Proteomics, 9(12), 3409-3412. doi:10.1002/pmic.200800646

Sigrist, C. J. a, Cerutti, L., de Castro, E., Langendijk-Genevaux, P. S., Bulliard, V., Bairoch, A., \& Hulo, N. (2010). PROSITE, a protein domain database for functional characterization and annotation. Nucleic Acids Research, 38(Database issue), D161-6. doi:10.1093/nar/gkp885

Weatheritt, R., \& Jehl, P. (2012). iELM - a web server to explore short linear motif-mediated interactions. Nucleic Acids Research, 40(Web Server issue), W364-9. doi:10.1093/nar/gks444

Zhang, J., Petit, C. M., King, D. S., \& Lee, A. L. (2011). Phosphorylation of a PDZ domain extension modulates binding affinity and interdomain interactions in postsynaptic density-95 (PSD-95) protein, a membrane-associated guanylate kinase (MAGUK). The Journal of Biological Chemistry, 286(48), 41776-85. doi:10.1074/jbc.M111.272583 


\section{Figure 1}

Flowchart of the typical workflow of DoReMi

1) The user typically provides the motif description in 3 different ways: Regular Expression(s) define a motif by the basic format allowed in most implementations. Amino acids are defined by their one-letter code with "." standing for any. Multiple potential residues can be encoded by using square brackets, e.g. "[DE]". To quantify selected residues, we allow the basic operators "*”, "+", “\{2\}” or "\{3,4\}”. To uses a PSSM for the motif search, the user simply provides a set of known binding motifs; these are used to calculate the amino acid distribution at each position, correcting for the overall amino acid distribution of the proteome. As a last option, users can provide results from other tools like SLiMsearch.

2) The second required input is the set of interacting domains. We provide the domains in PFAM-A. Proteins carrying any of the selected domains are defined as potential interaction partners. The highest scoring interacting protein for each motif instance is selected as potential binding partner.

3) The two scores are combined to rank each instance of the found motif. 


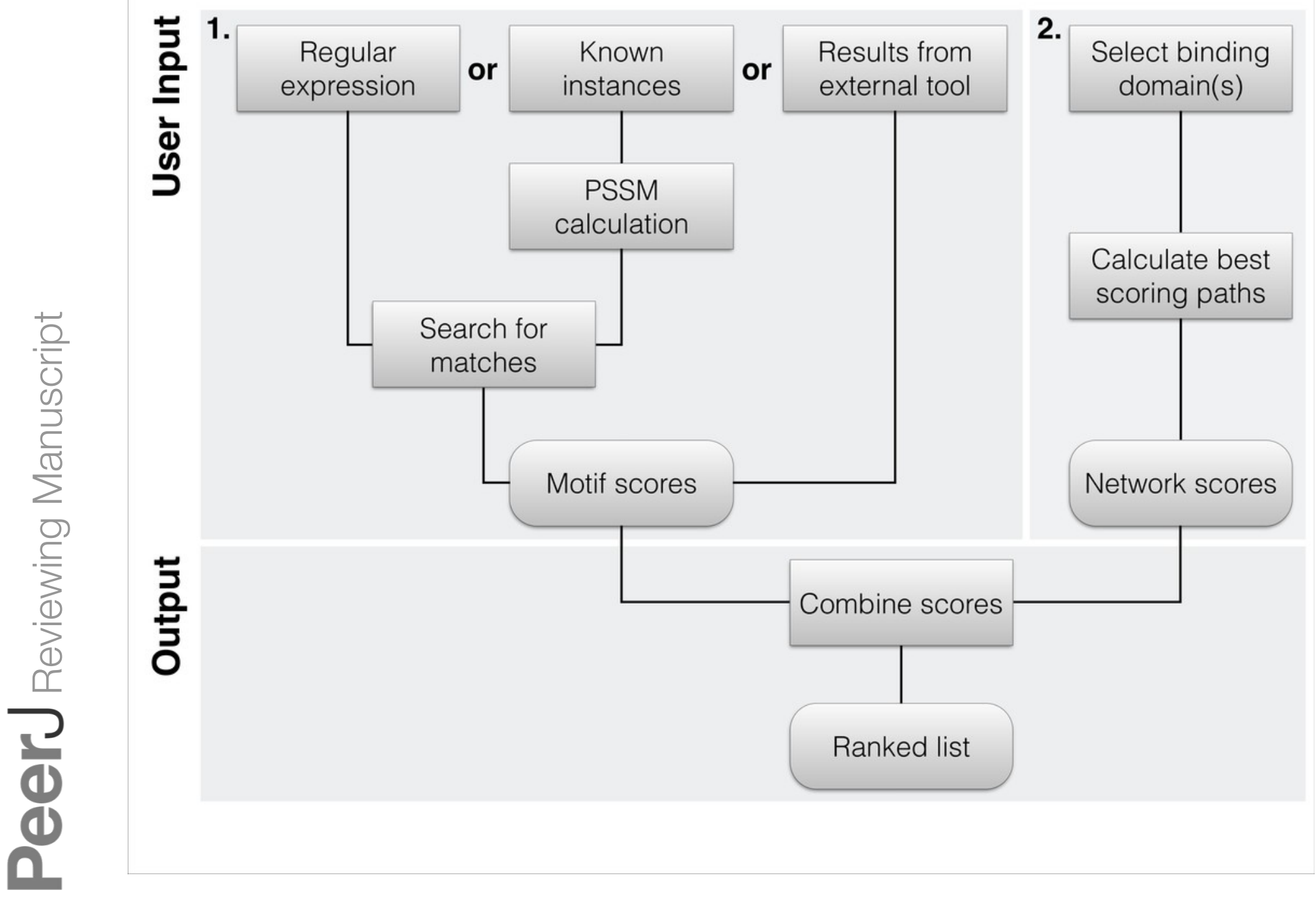




\section{Table 1 (on next page)}

Detailed performance numbers for the benchmarking.

The first number states the performance without contextual information while the second number includes contextual information. 


\begin{tabular}{|l|l|l|l|}
\hline & Sensitivity & Specificity & AROC \\
\hline SUMO - Split set - RegExp & $0.50 / 0.50$ & $0.98 / 0.99$ & $-/ 0.46$ \\
\hline SUMO - Split set - PSSM & $0.59 / 0.62$ & $0.96 / 0.96$ & $0.82 / 0.84$ \\
\hline SUMO - Partitioning & $0.75 / 0.77$ & $0.90 / 0.90$ & $0.82 / 0.84$ \\
\hline PDZ - Partitioning & $0.43 / 0.49$ & $0.90 / 0.90$ & $0.70 / 0.72$ \\
\hline
\end{tabular}




\section{Figure 2}

\section{Webinterface of DoReMi}

A) The interface allows the user to search for Pfam domains by their accession, id or description. From the list of search results, the user can select the relevant domains. If necessary, multiple searches can be performed to select differently named domains. B) The output page shows a brief summary of the analysis. This includes plots of score distributions for each score (motif, network and combined score) to aid in the selection of an appropriate score cut-off for downstream analysis of the results after downloading.

\section{A}

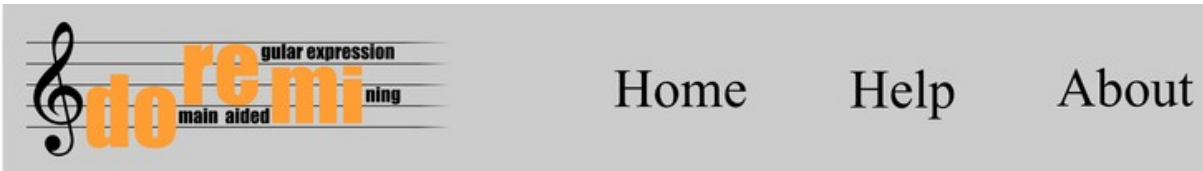

input motif data

select domain

view results

Select the domains of interest. \#1/2

Search by name SUMO

Found domains Rad60-SLD_2, Ubiquitin-2 like Rad60 SUMO-like

Search
Selected domains Rad60-SLD, Ubiquitin-2 like Rad60 SUMO-like

\begin{tabular}{ll} 
Domain & STRING hits \\
\hline Rad60-SLD & 53 \\
\hline URM1 & 1 \\
\hline Ub-RnfH & 1 \\
\hline HECT & 28 \\
\hline ubiquitin & 79 \\
\hline
\end{tabular}

Sort results by:

-Final Score $\bigcirc$ Network Score $\bigcirc$ Motif Score

Showing results 1 to 100

back next

Substrate name Substrate Id Position Domain protein name

CASC5

FAM9B

ENSP00000335463 513

ENSP00000378716 75

ENSP00000271764 255

\section{SUMO3} UHRF1

\section{$==>$ download results}

